\title{
Acapulco y el proyecto modernizador alemanista
}

Elmy Lemus Soriano ${ }^{1}$

elmygrisel@hotmail.com
Acapulco and the modernization project

alemanista

\section{Resumen}

El artículo analiza la construcción tanto urbanística como cultural del moderno puerto de Acapulco, en el marco del proyecto económico del periodo presidencial de Miguel Alemán. Asimismo, resalta el papel de la prensa nacional y extranjera, en la configuración exitosa de una ima- gen del puerto de Acapulco en la que se combinaba la idea de lo paradisiaco con lo moderno, misma que prevaleció hasta comenzar la década de los sesenta, cuando se comenzaron a evidenciar las fallas del proyecto económico mexicano.

Palabras clave: Acapulco, economía, modernidad, turismo, alemanismo.

\section{Abstract}

The article analyzes the configuration, as image of the port of Acapulco, that was much urbanistic as cultural, of the modern port of Acapulco, according to the economic project of the period of Miguel Aleman's presidency. It also emphasizes the role of the foreign and national press, in the successful configuration of a new combining the idea of the idyllic with the modern, the same prevailing until the beginning of the sixties, when the Mexican economic project showed an evident decrease.

Key words: Acapulco, economy, modernity, tourism, alemanismo.

1 Universidad Autónoma Metropolitana, Unidad Azcapotzalco, México. Avenida San Pablo 180, Del. Azcapotzalco, Col. Reynosa Tamaulipas, C. P. 02200, Ciudad de México, D. F. 
He paseado el mundo entero queriendo encontrar la gloria y puedo escribir la historia que es Acapulco, Guerrero: es un puerto natural, que le adornó su bocana y como él no hay otro igual en la nación mexicana. ${ }^{2}$

Al llegar Miguel Alemán Valdés a la presidencia de México ocurrieron importantes cambios en el ámbito nacional. En el aspecto económico, el proyecto alemanista tenía como prioridad la industrialización y el crecimiento acelerado del país, aprovechando los recursos generados durante la Segunda Guerra Mundial. Sin embargo, el hecho de que el capital mexicano no se encontrase todavía en condiciones suficientes para impulsar la economía nacional llevó al gobierno a emprender acciones que a simple vista podían parecer opuestas. Por un lado, se tomaron una serie de medidas proteccionistas con el fin de que las empresas mexicanas no se viesen obligadas a competir con los productos extranjeros; un discurso nacionalista que era incongruente con un capital nacional insuficiente para fortalecer la industria mexicana, por lo que muy pronto se tuvo que tomar la segunda medida, consistente en recibir al capital privado extranjero, el cual se vio favorecido por un mercado cerrado que no presentaba una fuerte competencia para sus productos. ${ }^{3}$ Si bien se impusieron restricciones al porcentaje de capital extranjero en ciertas ámbitos como el industrial, minero, agrícola, etcétera, en áreas como la industria de transformación, el comercio y los servicios las limitantes fueron menores. A la postre, las inversiones extranjeras, primordialmente estadounidenses, se concentraron precisamente en las últimas áreas mencionadas. ${ }^{4}$ En este panorama económico, Miguel Alemán y su grupo concibieron el turismo como una actividad económica prioritaria, ${ }^{5}$ en tanto que beneficiaba do- 
blemente al país, al atraer inversionistas nacionales y extranjeros, así como el dinero de turistas y viajeros, principalmente estadounidenses. Ya desde su campaña de gobierno, Miguel Alemán, apoyado por el economista Manuel Germán Parra, había organizado una serie de conferencias de mesa redonda para conocer las necesidades de los empresarios de todo México. En concreto, en el caso de Acapulco, durante las conferencias organizadas en octubre de 1945 se había subrayado la importancia de la actividad turística, la cual debía ser impulsada por el gobierno mexicano a través de distintos programas. En primer lugar, los empresarios propusieron la construcción y reparación de carreteras y hoteles; en segundo lugar, emprender un programa de publicidad y propaganda que motivase la elección de México, tomando como base la "política de buena voluntad" hacia los Estados Unidos imperante en la época de la Segunda Guerra Mundial, que había conciliado los intereses de nuestro país con los de los vecinos. Un tercer punto se refería a la capacitación de los trabajadores del sector para dar un servicio que pudiese competir con el de otros países. Finalmente, se proponía también que se concedieran vacaciones a los trabajadores del sector público y privado durante los meses de abril a octubre, época en la cual existía poco turismo extranjero. Los empresarios también solicitaban que se les otorgasen créditos con bajo interés, al mismo nivel que en el sector industrial, y que se les eximiera del pago de impuestos por al menos cinco años. Por su parte, Miguel Alemán se comprometía a fortalecer el turismo tomando en cuenta sus propuestas, lo que beneficiaría a México no sólo en materia económica sino también en el aspecto internacional, al estrechar las relaciones con Estados Unidos "fortaleciendo con el trato directo, la comprensión y la amistad de los ciudadanos la comunidad de ideales democráticos que se ha establecido entre ambas naciones". ${ }^{6}$

Por lo tanto, para dar continuidad a estos proyectos en el sector turístico, durante el periodo presidencial de Miguel Alemán se crearon la Dirección Nacional de Turismo, ${ }^{7}$ la Escuela Mexicana de Turismo y la primera Ley Federal sobre la materia. ${ }^{8}$ Miguel Alemán diría en la década de los sesenta, como presidente del Consejo Nacional de Turismo: "El turismo significa para el país receptor un ingreso considerable. Personas que han trabajado en otras partes vienen a consumir en él una parte de

6

8 Romero, Enciclopedia mexicana, v. IV, p. 68. Secretaría de Gobernación.

"El problema de la industria nacional del turismo", p. 45 en Archivo General de la Nacional (en adelante AGN), Presidentes, Miguel Alemán Valdés, exp. 545.3/239.

Anteriormente sólo se contaba con el Departamento de Turismo, dependiente de la 
sus ahorros. Eso supone adquisición de divisas, es decir, de medios de pago en el exterior". ${ }^{9}$

Por supuesto que el hecho de que Europa se encontrase destruida como resultado de la Segunda Guerra Mundial contribuía a que el turismo extranjero, principalmente del norte del río Bravo, buscase en México un nuevo destino para vacacionar. ${ }^{10}$ Para hacer del turismo una actividad económica prioritaria del país era necesario, como ya lo habían advertido los empresarios, desarrollar no sólo una fuerte infraestructura, vías de comunicación y hoteles de primera calidad, sino también una importante propaganda que pusiera a México en el imaginario turístico nacional y extranjero. Si bien se promovieron sitios como Zihuatanejo, Taxco y Cuernavaca, entre otros, fue Acapulco uno de los espacios que más cambios sufrió como resultado del proyecto alemanista de desarrollo turístico, tanto en el aspecto urbanístico como en el social y el económico. En ese sentido la prensa nacional fue de enorme importancia, pues a través de su apoyo y visto bueno al proyecto alemanista y a la transformación del puerto se logró convertir a Acapulco en un verdadero centro turístico de talla internacional. La llamada "Perla del Pacífico" devino símbolo del crecimiento nacional, por lo que las revistas del país y las extranjeras así como las guías turísticas de la década decidieron dedicarle un lugar especial.

Meses antes, sin embargo, el gobierno de Miguel Alemán había puesto en peligro la relación cordial con la prensa nacional, pues a tan sólo unos días de finalizar 1947 se había promulgado la nueva Ley sobre Derechos de Autor. En enero, los periódicos de mayor renombre en el ámbito nacional comenzaron a pronunciarse en contra de dicha ley, en especial de su artículo 7, pues prohibía cualquier publicación que fuese "en contra de la moral" o alterase el orden público. Al definirse tan vagamente, la ley se encaminaba a que el gobierno controlara a discreción aquello que considerase como contrario al orden, a la vez de permitirle censurar a su antojo. Esa ley sería nombrada por periódicos como La Prensa y el mismo Novedades "la ley mordaza". La principal consecuencia de su promulgación, en opinión del periódico La Prensa, era que

todo el sistema informativo de los periódicos queda sin defensa y el papel de investigador y censor de las costumbres, así como de orientador de la opinión pública, que representa un diario, queda a merced de cualquier malhechor que aproveche las armas que se le

\begin{tabular}{l|l}
9 & Alemán, Quince lecciones, p. 63. \\
10 & Romero, Enciclopedia mexicana, v. IV, p. 63.
\end{tabular} 
facilitan para defenderse contra los sostenedores del decoro y de la moralidad". ${ }^{11}$

Evidentemente, la prensa en ningún momento atacó de manera directa al presidente. Por el contrario, se dijo que la ley desvirtuaba el pensamiento de Alemán y era resultado de la aprobación apresurada e insensata del Congreso, que no deseaba perder sus vacaciones navideñas. ${ }^{12}$ Después de un acalorado debate en el que participaron la Asociación Nacional de Periodistas, el Sindicato Nacional de Redactores y el Secretario de Educación, Manuel Gual Vidal, la ley fue modificada en el mes de agosto eliminando el derecho de las autoridades a censurar las obras contrarias a la moral y negándoles únicamente el derecho de autor, ${ }^{13}$ lo que calmó los ánimos. A partir de entonces la prensa sería un arma propagandística sumamente provechosa para el régimen en términos políticos y económicos. A su vez, la prensa se vería beneficiada por las inserciones pagadas por los gobiernos federal y de los estados, a pesar de que éstas condicionasen en gran medida las críticas posibles al régimen. Tres años más tarde, Alemán instituyó el 7 de junio como el Día de la Libertad de Expresión y Prensa, motivo de reunión anual con los periodistas en la que el régimen restablecía su compromiso de mantener la libertad de expresión. ${ }^{14}$

\section{Acapulco}

Si bien desde 1927 se había dotado al puerto de una nueva carretera ${ }^{15}$ y un aeropuerto capaces de acortar el tiempo de traslado de la ciudad de México, fue a partir de 1948 que Acapulco se transformaría en un lugar turístico preponderante. Ese año el presidente emprendió una serie de obras que lo dotaron de una imagen acorde a la modernización alemanista, con la colaboración de empresarios nacionales y extranjeros y de

15

De hecho, la carretera a Acapulco formó parte del primer plan de la Comisión de
Caminos creada por Calles en 1925. No obstante, el viaje a Acapulco en aquella
época tenía una duración de aproximadamente 17 horas. Véase Waters, "Remapping
Identities", p. 222; Romero, Enciclopedia mexicana, v. IV, p. 18.

¡Caerá la mordaza!”, La Prensa, 20 de enero de 1948, primera sección, p. 8.

“¡Caerá la mordaza!”, La Prensa, 20 de enero de 1948, primera sección, p. 8.

"El nuevo texto para la libertad de prensa", La Prensa, 7 de septiembre de 1948, primera sección, p. 3.

Manuel Mendoza Zarabia, “Nuestros propósitos”, Armas, núm. 107, 20 de junio de 1951, p. 3 
la Junta Federal de Mejoras Materiales del puerto. El Secretario de Hacienda, Ramón Beteta, nombró a Melchor Perusquía para presidirla, ${ }^{16} \mathrm{y}$ estaba integrada por el presidente municipal del puerto y representantes de la Secretaría de Bienes Nacionales, del gobierno del estado de Guerrero, de la Secretaría de Salubridad, de la Cámara Nacional de Comercio, de la Secretaría de Comunicaciones y de la Secretaría de Marina. ${ }^{17}$ Para obtener los fondos necesarios a la remodelación de Acapulco, el gobierno federal expropió ejidos en buena parte de la zona costera (El Jardín, Progreso, El Marqués, Santa Cruz, La Garita y Las Cruces) y transfirió su propiedad a la Junta, la cual, a través de la Comisión Administradora de Terrenos de Acapulco, fraccionó y vendió los terrenos. Los ejidatarios fueron reubicados, la mayoría en los montes aledaños a la costa; se les construyeron modestas casas y recibieron diez mil pesos para que comenzaran un negocio, ya que su anterior modo de subsistencia agrícola había desaparecido.

Cada ejido expropiado respondió a un proyecto urbanizador distinto: El Marqués, por ejemplo, fue subdividido entre una amplia zona residencial para la construcción de hoteles de lujo y otra concebida como un conjunto de granjas que deberían resolver el problema del abastecimiento de granos que padecía Acapulco. En El Progreso, en cambio, se estableció una colonia proletaria donde el metro cuadrado fue vendido a razón de 4 pesos. ${ }^{18} \mathrm{El}$ dinero obtenido con la venta de los terrenos sirvió para la urbanización y la construcción de un complejo sistema de abastecimiento de agua y de electrificación del puerto. Tan sólo entre 1947 y 1948 se construyó un nuevo malecón, las calles fueron asfaltadas (se abrió la gran avenida costera que lleva el nombre de Miguel Alemán), se dotó a la zona costera de luz, de un nuevo mercado y hasta de un nuevo aeropuerto capaz de recibir vuelos nacionales y extranjeros; se le proveyó, en fin, de infraestructura, todo ello con una erogación de más de 10 millones de pesos,$^{19} \mathrm{y}$ finalmente comenzó un programa de renovación de la carretera

16 Melchor Perusquía nació en Nuevo León en 1906. Apoyó la candidatura de Manuel Ávila Camacho en Coahuila en 1939, por lo que fue nombrado administrador de la aduana de Veracruz y presidente de la Junta Federal de Mejoras Materiales en ese puerto. Gracias a su amistad con Ramón Beteta y con Miguel Alemán, cuando en 1945 se elaboró el plano regulador de Acapulco, Perusquía fue transferido a la Junta Federal de ese puerto. Adame, Obra y semblanza, p. 2.

Adame, Obra y semblanza, p. 14.

Adame, Obra y semblanza, pp. 103-105.

"Acapulco, lo nuevo y lo eterno", Acapulco. Revista Mexicana del Sur, 15 de julio de 1949, p. 2; Novedades, 21 de enero de 1948, p. 1. 
México-Acapulco. ${ }^{20}$ Para 1951, la suma invertida en Acapulco ascendía ya a 45 millones e incluía también la construcción de escuelas, hospitales y estacionamientos para los turistas. ${ }^{21}$

Entre los proyectos que nunca se concretaron se encontraba el establecimiento de hoteles de clase mundial que contasen con casinos. Para ello, Melchor Perusquía hizo una gira de tres meses por Europa, para finalmente proponer el Proyecto Acapulco. Asimismo, la Fraccionadora de Terrenos del empresario Alejandro Romero propuso una alianza con la naviera Grace Line que consistía en el establecimiento de una ruta de crucero con destino a un hotel de lujo con casino en la zona costera. Ambos proyectos fueron presentados al presidente, principalmente por los nexos de sus promotores con el grupo cercano a Alemán, específicamente con su secretario particular Rogerio de la Selva, a pesar de que la Ley de Juegos y Sorteos de 1947 prohibía la operación de casinos en México. Al parecer Adolfo Ruiz Cortines desempeñó un papel decisivo en el rechazo de esos proyectos, pues como Secretario de Gobernación decidió no otorgar los permisos correspondientes; en todo caso, ambas iniciativas dan cuenta del empeño por hacer de Acapulco un destino turístico internacional, así como del modus operandi de algunos funcionarios del momento. ${ }^{22}$

Por otro lado, con el fin de fomentar el turismo extranjero, se instaló una Oficina de Turismo mexicano en Nueva York y el vuelo directo desde esa ciudad a Acapulco; para decenas de veteranos de guerra, el puerto se convertiría además en un lugar de retiro. A partir de entonces, cada obra que se iniciaba en Acapulco fue encaminada a su desarrollo como centro turístico; por ejemplo, la construcción de la avenida costera Miguel Alemán embelleció la zona, pero eliminó la posibilidad de hacer las obras portuarias necesarias para la carga y descarga de buques de gran calado $\mathrm{y}$, con ello, cualquier posibilidad de convertirlo en un punto obligado del tráfico marítimo intercontinental. ${ }^{23}$

Sin embargo, el desarrollo material no era suficiente para colocar a Acapulco en el centro del turismo internacional, por lo cual la misma Junta de Materiales contempló un fuerte programa propagandístico que contribuyese a crear una imagen ideal de Acapulco y de lo que el puerto significaba en el gran programa modernizador de Miguel Alemán. En

\footnotetext{
20 p. 19.

Sotomayor, "Aquí Acapulco...", Novedades, 17 de junio de 1950, segunda sección, p. 7.

"Mejoras en Acapulco", "Nuestra República", Tiempo. Semanario de la vida y la verdad, vol. XIX, núm. 500, 30 de noviembre de 1951, pp. 21-22. AGN, Presidentes, Miguel Alemán Valdés, exp. 549.3/59, 15 f.

"La nueva calzada costera", Acapulco. Revista Mexicana del Sur, 1ํ de agosto de 1949,
} 
1949, el Segundo Congreso del Sindicato de Trabajadores de la Educación tuvo lugar en el puerto de Acapulco y coincidió con la visita del presidente Alemán. Estos hechos fueron presentados por la prensa local como de enorme trascendencia, pues permitían atraer la atención del país hacia Acapulco, que por fin había alcanzado "el lugar que por sus bellezas naturales y su clima delicioso le correspondía". ${ }^{24}$ Ese año, el Comité Coordinador de Turismo del Estado (compuesto por la ya mencionada Junta de Mejoras Materiales, el presidente de la Asociación de Hoteleros de Acapulco y los empresarios de la zona) informaba haber gastado más de doscientos mil pesos en inserciones en los periódicos más importantes de la República, tales como Excelsior, Novedades, El Universal, así como en el Anuario Turístico de la Asociación Nacional Automovilística, edición bilingüe que se distribuía en uno y otro lado de la frontera. ${ }^{25} \mathrm{La}$ distribución había sido la siguiente:

\begin{tabular}{|lcc|}
\multicolumn{1}{|c}{ Periódico } & Núm. de págs. & Monto desembolsado \\
\hline Excelsior & 48 & $\$ 78,400$ \\
Novedades & 19 & $\$ 30,400$ \\
El Universal & $7 \frac{1 / 2}{2}$ & $\$ 13,000$ \\
La Prensa & 9 & $\$ 9,000$ \\
El Nacional & 6 & $\$ 6,000$ \\
Gráfico & 2 & $\$ 2,000$ \\
Noticias & 3 & $\$ 4,000$ \\
Esto & 12 & $\$ 12,000$ \\
Mañana & 24 & $\$ 24,000$ \\
Hoy & 23 & $\$ 23,000$ \\
Nosotros & 9 & $\$ 9,000$ \\
México en Marcha & 9 & $\$ 9,000$ \\
Por Qué & 10 & $\$ 6,000$ \\
Nuevo Mundo & 6 & $\$ 6,000$ \\
Tiempo & 3 & $\$ 3,000$ \\
La Semana & 4 & $\$ 2,400$ \\
Todo & 8 & $\$ 6,400$ \\
Orientación & 8 & $\$ 4,800$ \\
México en la Mano & 20 & $\$ 10,000$ \\
Anuario Turístico AMA & 12 & $\$ 3,600$ \\
Continente & 1 & $\$ 1,000$ \\
\hline
\end{tabular}

\footnotetext{
24 "La gira presidencial", Trópico, 1ํ de marzo de 1949, p. 2.

25 "Más de medio millón en propaganda", Acapulco. Revista Mexicana del Sur, 1ํ de diciembre de 1949, p. 3.
} 
De igual manera, se comenzó a publicar una revista local llamada Acapulco. Revista Mexicana del Sur, editada quincenalmente por Xavier Campos Ponce, continuación de Palpitaciones Porteñas, revista publicada durante cinco años. En ellas no sólo se daba cuenta de la transformación de la zona, pues se incluían también cuentos, poemas y fotografías que contribuían a crear una imagen de Acapulco a la medida del proyecto alemanista:

Acapulco se ha normalizado como ciudad, sin haber perdido grandes aspectos de comodidad y belleza para los bañistas. Tiene ya su nuevo malecón [...] sus calles principales asfaltadas; sus calzadas de comunicación en condiciones de tránsito; agua abundante [...] luz, en raudales. ${ }^{26}$

Finalmente, el periódico local Trópico, dirigido por Manuel Pérez Rodríguez, publicó una serie de entrevistas hechas por su gerente, Carlos Adame, a Melchor Perusquía, presidente de la Junta Federal de Mejoras, en que se mostraban los enormes beneficios económicos y sociales que las obras darían a Acapulco, mencionando incluso los nombres de los ejidatarios a quienes se había indemnizado por las expropiaciones y respaldando, en fin, el proyecto de Miguel Alemán, "el hombre que lleva el timón de la patria por tan elevados senderos de progreso". ${ }^{27}$

Respecto a la prensa nacional, las inserciones pagadas por el Comité Coordinador del Turismo de Guerrero iban desde simples carteles con fotos del puerto hasta dossiers con el tema de Acapulco, o bien secciones especiales como la llamada "Aquí Acapulco", que se comenzó a publicar en 1950 en Novedades. Adicionalmente, los hoteleros y las empresas constructoras se anunciaban constantemente en los periódicos mencionados.

Entre las inserciones destaca un texto en especial, redactado por el periodista Carlos Villenave para Novedades. El artículo, intitulado "Acapulco: cielo, mar y mujeres", desplegado en dos páginas, daba cuenta del desarrollo del puerto:

Acapulco ya es un centro turístico completo, pues reúne a la esplendidez de sus paisajes de ensueño y la munificencia de sus playas grandes hoteles de primerísima categoría, a la altura o mejores que los de Europa y Estados Unidos. ${ }^{28}$

26

27

28

(

Acapulco. Revista Mexicana del Sur, 15 de julio de 1949, p. 1.

Adame, Obra y semblanza de un hombre, p. 97.

Villenave, "Acapulco: cielo, mar y mujeres", Novedades, 4 de marzo de 1949, segunda sección, p. 6.

Letras Históricas / Número 14 / Primavera-verano 2016 / México / pp. 211-231 / ISSN: 2007-1140 
El autor ponía énfasis en el compromiso del periódico con "el sacrificio que el Gobierno actual y la iniciativa privada han estado llevando al cabo en pro del mejoramiento de ese bello lugar", lo cual justificaba la extensa descripción de las obras en el puerto, sin hablar, por supuesto, de la enorme cantidad de dinero que la Junta Federal de Mejoras Materiales había pagado. En este sentido, era evidente el viraje en la forma en la que se presentaba Acapulco. Ocho años antes, en 1941, Excelsior había publicado un especial intitulado "Los más famosos lugares de México para vacaciones". Ahí figuraban Morelia, Guadalajara, San Luis Potosí, Torreón, Manzanillo, y tan sólo de manera breve Acapulco, del que se ensalzaba únicamente el paisaje, "sorprendente por su incomparable hermosura" ${ }^{29}$ En efecto, conforme avanzaba la década de los cuarenta, y principalmente con la llegada de Alemán al poder, el énfasis sobre las virtudes de los sitios turísticos nacionales era puesto en la modernidad -entendida como urbanización- del país que, combinada con sus tradiciones, hacía de México el lugar ideal para vacacionar..$^{30}$

Pero volviendo al año de 1949, habría que decir que inclusive la Asociación de Hoteleros de Acapulco decidió lanzar su propia publicación. Acapulco la ruta de oro del Turismo o Acapulco's Golden Route, publicación bilingüe que de manera mensual daba cuenta de los últimos acontecimientos del puerto, pero sobre todo de las tarifas y la ubicación de cada uno de los hoteles. En ese sentido, Acapulco devino el sitio a donde podían acudir lo mismo la naciente clase media mexicana que la clase política o el actor más famoso de Hollywood; todos se daban cita en el Pacífico mexicano; el puerto correspondía al proyecto de Miguel Alemán en la medida en que se demostraba que los beneficios económicos llegarían paulatinamente a todas las clases sociales. Desde entonces, Acapulco ofrecía una opción para cada bolsillo; inclusive para fomentar la visita al puerto la Asociación de Hoteleros, de la mano de su abogado Guillermo Lombardo Toledano, hermano de Vicente, creó el llamado Fondo de Vacaciones por medio del cual los ciudadanos podían adquirir títulos que se pagarían en abonos durante todo el año y que podrían ser utilizados en cualquier hotel cuando decidiesen visitar el puerto; los títulos iban desde los 100 hasta los 500 pesos, según el presupuesto del cliente, y los gastos ocasionados por su administración eran absorbidos por la Asociación. ${ }^{31}$

\footnotetext{
29 "Acapulco es sitio ideal para pasear", Excelsior, 4 de abril de 1941, tercera sección, p. 9.

30 Berger, The Development, p. 91.

31 Sanchis, "Acapulco ha dejado de ser privilegio de algunos para tornarse en recreo de muchos", Novedades, 6 de marzo de 1949, p. 10.
} 
El cliente, además, podía resultar ganador en alguno de los sorteos mensuales, con lo cual podría hacer uso del total de su bono aun antes de pagarlo. ${ }^{32}$

Con medidas como ésta se perfilaba una imagen de Acapulco al alcance de todos los bolsillos, aunque la realidad solía ser muy diferente. En la bahía más amplia se encontraban hoteles como los Bungalows Los Virreyes o la Pensión Granada, de plan europeo (sin comidas) y pensados para la clase media. En cambio, los hoteles más exclusivos, como Reforma Casablanca, Los Flamingos y El Mirador, fueron construidos en cerros y acantilados, totalmente separados de la avenida costera Miguel Alemán, con plan americano (que incluía las comidas) y hechos para el turismo extranjero. Equipados hasta con discotecas, no permitían el desarrollo del comercio local, dado que el visitante no solía dejar las instalaciones del hotel. ${ }^{33}$

En fin, la propaganda rendía frutos, pues para la Semana Santa de 1949 los periódicos capitalinos calculaban en más de 17 mil los visitantes a Acapulco, ${ }^{34}$ y para junio de 1951 Novedades anunciaba que tan sólo en ese mes 6048 paseantes nacionales y 5404 extranjeros habían visitado el puerto. Entre ellos destacaban Dolores del Río, la bailarina Tongolele y el cineasta Ismael Rodríguez. ${ }^{35}$

Finalmente, en 1951, el empresario y novelista Francisco Peláez Vega, cuyo seudónimo era Francisco Tario, publicó el libro Acapulco en el sueño, con fotografías de Lola Álvarez Bravo, al parecer a petición del mismo Melchor Perusquía. En su libro, dedicado a Miguel Alemán, Tario retrataba su experiencia personal de Acapulco como dueño de salas de cine, como empresario y como novelista. Respecto de la vida económica, afirmaba que Acapulco

produce espuma, exporta raros y oscuros romances [...] su principal fuente de vida es la vida misma, y su penuria mayor, la muerte [...]. Potencialmente hablando, trátase a grandes líneas de un centro vital de excepcional importancia. ${ }^{36}$

\footnotetext{
32 Folleto "Acapulco... paraíso tropical” en AGN, Presidentes, Miguel Alemán Valdés, exp. $548.2 / 12$.

33 Sackett, "The two faces of Acapulco", p. 504.

34 "El regreso de los vacacionistas no va a ser tan fácil", Novedades, 17 de abril de 1949, p. 1.

35 Sotomayor, "Aquí Acapulco", Novedades, 12 de julio de 1951, segunda sección, p. 7.

36 Tario, Acapulco en el sueño, p. 54.
} 
¿Cuál fue entonces la imagen que logró atraer a propios y extraños? Emulando el proyecto alemanista, Acapulco sería en ese sentido promocionado como el lugar de ensueño en el que se combinaban los tres elementos del México posrevolucionario. En primer lugar, era un puerto de origen colonial, es decir, en sus calles se respiraban la tradición y la historia mexicanas. En segundo lugar, era un espacio tropical, con las implicaciones sensuales que eso conlleva: lugar de desinhibición, de bellos atardeceres y fiesta nocturna. Finalmente, Acapulco era además un lugar absolutamente moderno, sus construcciones podían competir con las de Miami o de cualquier otro puerto estadounidense o europeo. Tanto así que estrellas de todo el mundo acudían a observar con sus propios ojos uno de los ejemplos más claros del desarrollo mexicano. Si la Ciudad Universitaria era el monumento de la modernización alemanista en la ciudad de México, Acapulco lo era junto al mar.

De un pueblo rústico con bellas playas, hace veinte años, Acapulco se ha convertido ahora en un puerto de primera magnitud. Toma la forma de los puertos modernos y su primer malecón comienza a dar sus frutos, al venir siendo terminados por quienes realizan las obras portuarias. ${ }^{37}$

La conversión del puerto en lugar turístico por excelencia transformó profundamente la vida de sus habitantes en más de un sentido. Geográficamente, los pobladores se vieron obligados a dejar sus tierras cercanas a la costa, las cuales fueron invadidas por decenas de compañías constructoras que, al más puro estilo alemanista, pertenecían a importantes funcionarios como el Secretario de la Defensa Nacional, general Gilberto Limón, Manuel Ávila Camacho o el mismo Miguel Alemán; tan sólo para finales de 1948 se habían proyectado al menos 600 casas en la zona costera. ${ }^{38} \mathrm{La}$ indemnización a las familias afectadas -que en algunas casos incluyó una casa a las afueras de Acapulco- no cubría el daño a las actividades económicas, mucho menos el deterioro ecológico que comenzaba a perfilarse. ${ }^{39}$ En el aspecto demográfico, la población de Acapulco aumentó considerablemente en menos de una década: mientras que para

\footnotetext{
37 Acapulco, Revista Mexicana del Sur, 15 de julio de 1949, p. 2.

38 "Antes de terminar 1948 habrá 600 nuevas casas en Acapulco", La Prensa, 14 de enero de 1948, p. 13. Para 1951 solamente 2 de los 45 millones invertidos en Acapulco habían servido para indemnizar a las familias afectadas. "Mejoras en Acapulco", "Nuestra República", Tiempo, semanario de la vida y la verdad, 30 de noviembre de 1951, pp. 21-22.
} 
1940 la única localidad que rebasaba los diez mil habitantes en Guerrero era Iguala, para 1950 la población de Acapulco ascendía a poco más de 34 mil habitantes, sin contar los municipios aledaños. ${ }^{40}$ Finalmente, un programa modernizador como el puesto en marcha por Miguel Alemán incluía que los pobladores de Acapulco tuvieran que modificar sus hábitos y conductas; por ejemplo, se prohibió que burros y caballos anduviesen sueltos por la avenida costera Miguel Alemán, ya que solían acabar con el pasto y las plantas del camellón; se emprendió un programa para acabar con los perros callejeros y se exigió a los habitantes del puerto mantener aseados los frentes de sus casas, de lo contrario se harían acreedores a una multa de entre 5 y 25 pesos, según el caso. ${ }^{41}$ De igual manera, los hoteleros exigían a las autoridades mayor control sobre las líneas de autobuses locales, las cuales permitían que los choferes y sus cobradores cobrasen tarifas excesivas en autobuses sucios y en malas condiciones. ${ }^{42}$ A pesar de los esfuerzos para mantener el puerto a la altura de las expectativas internacionales, un turista comentaba en 1949:

el paseante busca una banca para descansar y las pocas que hay están rotas y sucias, encima de ellas hay cáscaras de plátano, de naranja, chorreaduras de paletas y hasta alimentos devueltos del estómago por los borrachos y mareados que adornan esos escasos asientos. ${ }^{43}$

Con la llegada de Ruiz Cortines a la presidencia se promovió también la reducción de tarifas en el hospedaje, con el fin de fomentar el turismo nacional que había decaído con la devaluación del peso en 1953. En el mismo sentido, se organizó el Primer Congreso Nacional de Turismo Interior, donde se hicieron distintas propuestas para garantizar que los mexicanos conocieran el puerto de Acapulco, así como para fomentar la redacción de leyes estatales para el turismo que correspondiesen a la ley federal hecha en el sexenio anterior ${ }^{44}$ La prensa nuevamente contribuyó a este proyecto organizando en Acapulco la octava Convención Nacional del Sindicato de Redactores de Prensa, alabando el buen trato que se les había dado en el puerto y criticando a aquellos periódicos capitalinos que se atrevían a poner en duda los servicios del lugar. En efecto, para 1953 no faltaban

\footnotetext{
40 Sotomayor, “Aquí Acapulco...", Novedades, 13 de junio de 1950, segunda sección, p. 2. 
comentarios en la sección "Aquí Acapulco" de Novedades sobre abusos en las tarifas de hoteles y taxis así como de asaltos a turistas, lo que daba cuenta de la realidad que se vivía en el puerto, aunque también podía deberse al hecho de que Ruiz Cortines no siguió el programa de inserciones, publicidad y propaganda que había emprendido Miguel Alemán. ${ }^{45}$

\section{La mirada internacional}

El esfuerzo coordinado entre autoridades y empresarios por poner a Acapulco en el centro del turismo comenzó a rendir frutos cuando las guías turísticas internacionales decidieron ocuparse del puerto sureño. Como veremos, la mirada extranjera se debatía entre lo exótico de la cultura, lo moderno del México alemanista y lo paradisiaco de Acapulco.

Por ejemplo, en el mes de abril de 1947 la revista Time dedicaba un artículo al presidente de México. Con el sugerente título de "Good Friend", el texto daba cuenta del proyecto económico de Miguel Alemán, cuyo presupuesto, según el artículo, era el fin de la Revolución mexicana y el principio de la modernización del país. Evidentemente dicha tarea no podría ser llevada a cabo sólo por los mexicanos, y por ello se invitaba a los estadounidenses a invertir en el país y apoyarlos. Después de dedicar algunas páginas a elogiar al presidente, se hacía una advertencia: "Después de cinco meses en la presidencia, Alemán no ha olvidado cómo relajarse y divertirse". En efecto, el presidente pasaba los fines de semana en Cuernavaca, o bien en la casa que Ramón Beteta tenía en Acapulco. Finalmente Miguel Alemán regresaba a trabajar el lunes por la mañana, pues según él "México tuvo su Revolución... Es tiempo de ir a trabajar". ${ }^{46}$ En efecto, el hecho de que Miguel Alemán hiciese continuos viajes de descanso al puerto dotó a éste de un glamour extra, el presidente era la propaganda en sí misma y Acapulco era el sitio en donde Alemán paseaba en yate ${ }^{47}$ practicaba el golf y vacacionaba en su magnífica residencia ubicada en Puerto Marqués, "de un piso... con techos pintados de un verde vivo". ${ }^{48}$

De esta forma, la propaganda mexicana se veía rebasaba favorablemente por la extranjera. No sólo revistas como Harper's o National Geographic dedicaron múltiples páginas a la promoción del lugar paradisiaco, sino que celebridades como Cary Grant, John Wayne o el mismo presi-

\footnotetext{
45 AGN, Presidentes, Adolfo Ruiz Cortines, exp. 548/29.

46 "Good Friend", Time, Lunes 28 de abril de 1947.

47 Sotomayor, "Aquí Acapulco...", Novedades, 12 de julio de 1951, segunda sección, p. 7.

48 "Vacaciones de don Miguel", Tiempo, 8 de junio de 1951, p. 3
} 
dente John F. Kennedy con su esposa tomarían vacaciones en el puerto sureño. ${ }^{49}$ Este lugar central de Acapulco en el turismo de la década de 1950 es evidente en las continuas menciones de guías turísticas escritas por estadounidenses sobre nuestro país. Por ejemplo, en 1959 el profesor de español y de Filosofía en la Universidad de California, John A. Crow, publicó su libro intitulado Mexico Today. El libro de Crow, a medio camino entre la recuperación histórica y la guía de viajes, definía a México como una nación que, a través del mestizaje, había formado una "fascinante y única cultura moderna", ${ }^{50}$ una cultura "más extraña que Europa". ${ }^{51} \mathrm{El}$ autor, quien aseguraba conocer México profundamente, consideraba que Acapulco era un centro urbano de la misma importancia que las ciudades de México, Monterrey y Guadalajara, las cuales habían adquirido un "aire de modernidad y pulcritud" que les faltaba hacía veinte años. En tanto que el gobierno era estable y la economía sólida, estos sitios eran muestra de la prosperidad del país. "La gente de México vive mejor, come mejor y se siente mejor que nunca antes en su historia". ${ }^{52}$ Sin embargo, no todo era mérito nacional; Crow aseguraba que parte de la modernidad acapulqueña se debía, sin duda, a la llegada masiva de estadounidenses a la costa.

En el mismo sentido se expresaba John Wilhelm en su libro Guide to All Mexico, publicado en 1959. El autor, quien afirmaba haber vivido seis años en nuestro país, hacía, al igual que Crow, un recuento histórico para convencer al lector de la utilidad de su guía de viaje. Wilhelm dedicaba un capítulo de los quince que componen su publicación al puerto de Acapulco; la primera frase, "Acapulco está en el trópico", ${ }^{53}$ da el cariz de las 25 páginas dedicadas al puerto. El autor define a Acapulco como una de las diez mejores playas en el mundo y lo defiende de quienes lo consideran "muy comercial" o "con demasiados americanos". Para Wilhelm, esas características hacían de Acapulco un lugar paradisiaco y radicalmente opuesto a las otras playas mexicanas del Pacífico; "es mejor desayunar junto a una hermosa alberca que pasar el tiempo espantando las moscas que se acercan a tu comida", decía el autor. En cuanto a la cantidad de estadounidenses, el autor simplemente argumentaba que "Acapulco les gusta tanto como a mí". ${ }^{54}$ Sin duda, tal visión habría de cambiar hacia la década siguiente.

\footnotetext{
49

53 Wilhelm, Guide to All Mexico, p. 45.

54 Wilhelm, Guide to All Mexico, p. 47.

Niblo, "Acapulco in Dreams and Reality", p. 44.

Crow, Mexico Today, p. 1

Crow, Mexico Today, p. XIII.

Crow, Mexico Today, p. 305.
} 
El cine también contribuyó a la creación de ese imaginario acapulqueño. Entre 1950 y 1970 se realizaron diez películas cuya trama principal se desarrollaba en Acapulco. Directores tan renombrados en la época como Emilio el Indio Fernández (Acapulco, 1951) y Alejandro Galindo (Sucedió en Acapulco, 1952) contribuyeron a proyectar la imagen de un puerto a la vez exótico y moderno, divertido y lleno de aventuras. Estados Unidos participaría también con las películas Fun in Acapulco (1963), protagonizada por Elvis Presley, Acapulco Spies (1977) y Acapulco Gold (1977).

\section{Hacia el desencanto}

Si tuviésemos que hacer una cronología, diríamos que el "amor" por Acapulco comienza a declinar en la década de 1960. Fue en esa época cuando el esfuerzo emprendido por Miguel Alemán demostró haber beneficiado solamente a unos cuantos, la mayoría extranjeros; la población acapulqueña, por el contrario, se había visto despojada de sus terrenos en la bahía, confinada a la periferia, sin recursos ni servicios. Ya dos años atrás, el exsecretario de la Defensa Nacional en el periodo de Miguel Alemán, el general Gilberto R. Limón, había sido acusado de fraude, pues junto con la Compañía Mexicana Constructora Rural había fraccionado y vendido 685 hectáreas en la zona de Icacos en Acapulco, las cuales por decreto presidencial habían sido otorgadas a los ejidatarios a finales del año de 1946. Limón se había hecho millonario gracias a la protección del gobierno de Miguel Alemán, quien nunca hizo ejecutar el decreto expedido por su antecesor. ${ }^{55}$

Un año después, Francisco de P. Carral, como representante de la Delegación Federal de Turismo en Acapulco, enviaba un informe al Departamento de Turismo en el cual solicitaba el apoyo de la presidencia y del ejército para resolver los problemas de Acapulco. En su solicitud hacía evidente el descenso del turismo en el puerto, tanto por los abusos en las tarifas de hoteles, yates y veleros, como por la existencia de "maleteros piratas", quienes extorsionaban a los hoteleros para que, a cambio de cierta tarifa, se encargasen de llevar a los turistas desde la central de autobuses hasta sus hoteles. En caso de que un hotelero no pagase la cuota, los "maleteros pirata" usaban inclusive la fuerza para obligar al turista a hospedarse en otro hotel. ${ }^{56}$ Proponía, por tanto, que algunos infantes de marina resguardasen los embarcaderos y que otros tantos militares custodiasen la terminal de autobuses para poner fin a ambos problemas.

\footnotetext{
55 “Gilberto R. Limón, acusado de fraude", Excelsior, 22 de mayo de 1958, p. 1.

56 AGN, Presidentes, Adolfo López Mateos, exp. 548/20.
} 
Antes de que su proyecto fuese aceptado, en noviembre de 1960, una auditoría especial en Acapulco demostró un déficit de dos millones de pesos, lo que colocó al puerto en un estado de emergencia que tuvo que ser remediado por el presidente Adolfo López Mateos, quien otorgó un préstamo directo para que se pudiese celebrar la tercer Reseña Cinematográfica, que tenía lugar anualmente. ${ }^{57}$

Con Miguel Alemán a la cabeza del Departamento de Turismo, se decidió retomar el proyecto de publicidad para atraer el turismo extranjero, esta vez específicamente en publicaciones internacionales y a través de la compañía llamada Panamericana de Publicidad, S.A. Según la propuesta aceptada por el Departamento, en 1960 se gastarían 400 mil dólares por publicidad en 22 revistas entre las que se encontraban Holiday, National Geographic, Life, Sunset y Harper's Magazine. Otros 300 mil dólares serían destinados a periódicos estadounidenses como el Herald Tribune. ${ }^{58}$ La empresa Eco Publicistas, por su parte, diseñó ese mismo año una campaña apoyada en la figura de Cantinflas para promover el turismo en Estados Unidos y Canadá.

Este esfuerzo, sin embargo, se vio seriamente entorpecido cuando comenzó el conflicto con el gobernador de Guerrero, el general Raúl Caballero Aburto, que llevaría a la crisis al puerto, pues las continuas manifestaciones en su contra, que culminarían con su renuncia en 1961, provocaron que disminuyera el turismo durante ese año. ${ }^{59}$ En efecto, el lugar paradisiaco, símbolo de la modernidad mexicana, había sido construido a costa del sistemático despojo de las tierras de los habitantes de la costa, quienes ya sin hogar se habían visto obligados a invadir tierras en La Laja en el año de 1958. Eso, no obstante, tampoco significó una mejora para estas familias, que muy pronto se vieron sometidas por Alfredo López Cisneros, alias el rey Lopitos -sobrino de Caballero Aburto-, quien asumiría el control de dichos predios y que con un grupo de matones otorgaba a discreción tierras, cortaba o suministraba agua y luz; en fin, fungía como cacique local. ${ }^{60}$ El rey Lopitos fue asesinado en 1966 por unos matones contratados por el entonces gobernador de Guerrero, Raymundo Abarca Calderón, con lo que terminaron ocho años de cacicazgo e impunidad en La Laja. ${ }^{61}$

\footnotetext{
57 "Déficit de dos millones en las arcas de Acapulco", Novedades, 6 de noviembre de 1960, primera sección, p. 11.

58 AGN, Presidentes, Adolfo López Mateos, exp. 548/10.

59 "Las fuerzas vivas de Acapulco se dirigen a Adolfo López Mateos", Novedades, 13 de noviembre de 1960, primera sección, p. 1.

60 Estrada, El movimiento anticaballerista, pp. 54-55.

61 Sackett, “The Two Faces of Acapulco", p. 508.
} 
En ese sentido, la literatura, capaz de reflejar la realidad por la vía de la ficción, fue la primera en mostrarse suspicaz y crítica frente a la imagen moderna que se había construido alrededor del puerto de Acapulco. En fecha tan temprana como 1952, José Mancisidor publicó un cuento intitulado "Chino", cuyo personaje principal era un pescador acapulqueño. La historia, que emulaba en algunos sentidos El extranjero de Albert Camus, narraba el asesinato cometido por el hombre apodado el Chino, proveniente de la zona más pobre de Acapulco. Un buen día, el protagonista encuentra y asesina en la playa a un estadounidense, un gringo que quiso humillarlo y que sólo logró hacerle consciente de los contrastes entre su viejo Acapulco, simple y pobre, y el nuevo: lujoso, artificial, pero sobre todo excluyente. ${ }^{62}$ Cuatro años más tarde, Luis Spota, en Casi el paraíso (1956), retrató de tal forma la vida de los políticos mexicanos y de la burguesía en el puerto, que la simulación y la doble moral adquirían preponderancia sobre la comodidad y el lujo propios de aquellas mansiones compradas con dinero proveniente de "la justicia de la Revolución". ${ }^{63}$ Por su parte, Carlos Fuentes, en La región más transparente (1958), retrataba también la vida de la clase política en Acapulco, pero la contrastaba fuertemente con la experiencia de aquella familia que, aun reuniendo todos sus ahorros, llegaba en busca de un motel barato, sabiendo que el resto del año pagaría la deuda adquirida con el viaje. ${ }^{64}$

Del otro lado de la frontera, a comienzos de la década de los sesenta la revista Time dedicaba sus páginas no a ensalzar, sino a criticar el alguna vez sitio de ensueño. La contaminación y la afluencia masiva de turistas empezarían a desdibujar el imaginario construido. En lugar de Acapulco, que "comenzaba a lucir como Miami Beach", se recomendaba visitar Puerto Vallarta, un lugar de auténtico descanso y sobre todo un sitio donde los turistas podrían conocer al "verdadero México". ${ }^{65}$

Por su parte, la revista Life publicaba un número especial sobre México en 1961, traducido al español un año después, en plena crisis por el movimiento anticaballerista. En poco más de 150 páginas se daba cuenta de las impresiones del autor William Weber Johnson. El balance general resultaba en un país lleno de contrastes, donde la justicia de la Revolución no había llegado aún a todos y en el que la democracia era poco me-

\footnotetext{
62 Mancisidor, "Chino", El Legionario, 17, julio de 1952, p. 7. Spota, Casi el paraíso, p. 41. Fuentes, La región más transparente, p. 369.

“Everybody's Hideaway”, Time, viernes 1ํ de noviembre de 1963.
} 
nos que inexistente. ${ }^{66} \mathrm{El}$ único párrafo dedicado a Acapulco parecía ser una síntesis de la idea general del libro:

El habitante de la ciudad y el del campo raras veces se mezclan en México. El campesino que desciende de su sencilla aldea montañosa a un centro de verano como Acapulco se ve convertido de pronto en un extranjero en medio de sus coterráneos. Entre la ciudad y su choza de adobe media un abismo de kilómetros y de siglos. ${ }^{67}$

Quizás uno de los golpes más duros fue el asestado a través de un artículo publicado en el año de 1964 por la revista National Geographic. Bajo el título de "The Two Acapulcos" o "Los dos Acapulcos", el periodista James Cerrutti entregaba una visión por demás crítica de lo que el desarrollo turístico había arrojado a las playas acapulqueñas. Por un lado, el Acapulco auténticamente moderno, el que se entregaba al turismo extranjero y que recibía fuertes dividendos por ello, y en contraste el otro Acapulco, aquél de los porteños sin empleo, sin recursos, inclusive sin servicios básicos y que se habían concentrado cerca de la zona costera, en La Laja. ${ }^{68}$

A la postre, no sólo Acapulco sería despojado de su halo de perfección. En 1969 Roger Hansen daba a conocer su libro La política del desarrollo mexicano, en el cual se evidenciaban todos los escollos del famoso y quizás inexistente "milagro mexicano": desarrollo económico, sí, pero desigual distribución de la riqueza; estabilidad política, pero a cambio de la represión y el autoritarismo. ${ }^{69}$

En conclusión, con la llegada de Miguel Alemán al poder, el turismo adquirió preponderancia entre las actividades económicas del país. Acapulco devendría el proyecto más ambicioso en el sentido de la creación de un sitio a la vez paradisiaco y ultramoderno. Acapulco fue una tierra que transformó su realidad para convertirse en la tierra de ensueño de

\footnotetext{
66 "La presidencia está investida de un poder autocrático que, por lo general, se ejerce benignamente, pero de un modo casi absoluto... el voto electoral del ciudadano [...] en la mayoría de los casos únicamente sirve simplemente para confirmar decisiones que ya previamente han sido debidamente aprobadas. Casi no existen organismos que, como en otros países, limiten y equilibren el poder Ejecutivo". Véase Biblioteca universal de Life, México, pp. 72-73. Cerrutti, "The Two Acapulcos", National Geographic, vol. 126, núm. 5, diciembre de 1964, p. 864.

69 Hansen, La política del desarrollo, pp. 113-128.
} 
propios y extraños y cuyos habitantes vivieron de cerca los contrastes de un Estado posrevolucionario que lo mismo podía propiciar políticos multimillonarios y estrellas de cine que campesinos despojados de tierras. En fin, Acapulco parece ser la metáfora viva del proyecto alemanista y del "milagro mexicano".

\section{Archivos}

AGN Archivo General de la Nación, Fondos Presidentes Miguel Alemán Valdés, Adolfo Ruiz Cortines y Adolfo López Mateos.

\section{Publicaciones periódicas}

Acapulco. La ruta de oro del turismo (1950-1951).

Acapulco. Revista Mexicana del Sur (1949).

El Legionario (1950-1951).

Excelsior (1945-1965).

Harper's.

La Prensa (1945-1965).

National Geographic (1945-1975).

Novedades (1945-1965).

Palpitaciones Porteñas (1949).

Time (1945-1975).

\section{Bibliografía}

Alemán Valdés, Miguel

Quince lecciones de turismo, México, Diana, 1983.

Anuario Turístico de México, México, Asociación Nacional Automovilística, 1947 y 1949.

Berger, Dina

The Development of Mexico's Tourism Industry. Pyramids by Day, Martinis by Night, Nueva York, Palgrave-Macmillan, 2006.

Brenner, Anita

Your Mexican Holiday. A Modern Guide, Nueva York, Putnam's Sons, 1932.

Cerrutti, James

"The Two Acapulcos", National Geographic, vol. 126, núm. 5, diciembre de 1964, pp. 848-878.

Crow, John A.

Mexico Today, Nueva York, Harper \& Brothers, 1957.

Estrada Castañón, Alba Teresa

El movimiento anticaballerista: Guerrero 1960. Crónica de un conflicto, Chilpancingo, Universidad Autónoma de Guerrero, 2001. 
Fuentes, Carlos

La región más transparente del aire, México, Alfaguara, 2008.

Hansen, Roger

La política del desarrollo mexicano, México, Siglo xxI, 2000.

Martínez, María Antonia

El despegue constructivo de la nación. Sociedad y política en el alemanismo, México, Centro de Investigaciones y Estudios Superiores en Antropología Social, Miguel Ángel Porrúa, 2004.

Martínez, María Antonia

"El modelo económico de la presidencia de Miguel Alemán", en Will Fowler (coord.), Gobernantes mexicanos, México, Fondo de Cultura Económica, 2008, v. II, p. 287-335.

Niblo, Stephen y Diane M. Niblo

"Acapulco in Dreams and Reality", Mexican Studies, vol. 24, núm. 1, invierno de 2008, pp. 31-51.

Romero, Héctor Manuel

Enciclopedia mexicana del turismo, 5 v., México, Actualización y comunicación en el turismo, 1972.

Sackett, Andrew

"The Two Faces of Acapulco During the Golden Ages", en Gilbert Joseph y Timothy Henderson, The Mexico Reader: History, Culture, Politics, Londres, Duke University Press, 2003, pp. 500-511.

Spota, Luis

Casi el paraíso, México, Debolsillo, 2004.

Villanueva, Onésimo

"Romance para Acapulco", Palpitaciones Porteñas, núm. 109, 1ํ de enero de 1949.

Waters, Wendy

"Remapping Identities: Road Construction and Nation Building in Posrevolutionary Mexico", en M.K. Vaughaun y Stephen E. Lewis, The Eagle and the Virgin. Nation and Cultural Revolution in Mexico, Durham y Londres, Duke University, 2006, pp. 221-242.

Weber Johnson, William

Biblioteca Universal de Life en español. México, México, Offset Multicolor, 1961.

Wilhelm, John

Guide to All Mexico, 5ª ed., Nueva York, Mc Graw-Hill, 1959.

Recibido: 12/09/2014. Aceptado: 05/05/2015 